04.2

\title{
Измерение температуры электронов плазмы токамака Глобус-М2 методом мультилазерного томсоновского рассеяния
}

\author{
(ㄷ Г.С. Курскиев ${ }^{1}$, Н.С. Жильцов ${ }^{1}$, А.Н. Коваль ${ }^{1}$, А.Ф. Корнев ${ }^{2}$, А.М. Макаров ${ }^{3}$, Е.Е. Мухин ${ }^{1}$, Ю.В. Петров ${ }^{1}$, \\ Н.В. Сахаров ${ }^{1}$, В.А. Соловей ${ }^{4}$, Е.Е. Ткаченко ${ }^{1}$, С.Ю. Толстяков ${ }^{1}$, П.В. Чернаков ${ }^{5}$ \\ ${ }^{1}$ Физико-технический институт им. А.Ф. Иоффре РАН, Санкт-Петербург, Россия \\ ${ }^{2}$ ООО „Лазеры и оптические системы“, Санкт-Петербург, Россия \\ ${ }^{3}$ Университет ИТМО, Санкт-Петербург, Россия \\ ${ }^{4}$ Петербургский институт ядерной физики им. Б.П. Константинова НИЦ „Курчатовский институт“, \\ Гатчина, Ленинградская обл., Россия \\ ${ }^{5}$ АО „Спектрал-Тех“, Санкт-Петербург, Россия \\ E-mail: Gleb.Kurskiev@gmail.com
}

Поступило в Редакцию 6 сентября 2021 г.

В окончательной редакции 20 сентября 2021 r.

Принято к публикации 20 сентября 2021 г.

\begin{abstract}
Диагностика электронного компонента плазмы методом томсоновского рассеяния лазерного излучения позволяет измерить динамику пространственных распределений температуры $T_{e}(R, t)$ и концентрации $n_{e}(R, t)$ электронов. Одним из препятствий реализации диагностики томсоновского рассеяния в термоядерных реакторах является искажение спектральной характеристики оптической системы вследствие радиационно-наведенного поглощения и загрязнения оптических элементов продуктами эрозии первой стенки. Вследствие этого достоверность измерений методом томсоновского рассеяния будет снижаться со временем. Дано описание метода мультилазерного томсоновского рассеяния, который позволит решить указанную проблему. Приводятся результаты первых экспериментов на токамаке Глобус-М2.
\end{abstract}

Ключевые слова: диагностика плазмы, токамак, томсоновское рассеяние, лазерная диагностика плазмы, спектроскопия.

DOI: 10.21883/PJTF.2021.24.51799.19019

Неотъемлемым требованием, предъявляемым к диагностическим системам томсоновского рассеяния (ТР) в реакторных условиях, является возможность измерения температуры электронов в предположении, что спектральная характеристика системы сбора света неизвестна и может меняться во времени [1,2]. Данная проблема решается с помощью метода мультиволнового лазерного зондирования [3]. Этот подход планируется применять во всех трех диагностических системах ТР реактора ИТЭР, включая системы диагностики центральной, краевой и диверторной плазмы $[2,4]$. Первые предварительные эксперименты по апробации данного подхода были выполнены на установках RFX-mod [5] и Глобус-М [6]. Метод основан на наблюдении сигналов томсоновского рассеяния от зондирующих лазеров, генерирующих излучение на разных длинах волн. Главным предположением является условие неизменности температуры электронов в области наблюдения, ограниченной пересечением зондирующего лазерного пучка и проекции на него изображения детектора, на временно́м интервале, определяемом задержкой между лазерными импульсами. В проведенных ранее экспериментах на установках Глобус-M и RFX-mod это условие не выполнялось, так как временно́й интервал между лазерными импульсами был сравним с характерными временами развития магнитогидродинамических неустойчивостей. Другой вариант данного подхода предполагает наблю- дение сигнала ТР из заданного объема плазмы, измеренного под двумя разными углами одновременно. Такой подход, позволяющий оценивать параметры плазмы, используя по-разному уширенные спектры ТР, был экспериментально протестирован на установке TST-2 [7]. Этот вариант имеет ряд существенных ограничений применительно к термоядерному реактору, поскольку требует использования либо сложной геометрии сбора рассеянного излучения, либо многопроходной зондирующей системы (предполагающей как минимум двукратное прохождение лазерного излучения через исследуемую область плазмы). Для реализации рассматриваемого подхода временно́й интервал между лазерными импульсами должен быть минимальным, желательно на временно́м масштабе, меньшем, чем характерные времена изменения температуры электронов.

В настоящей работе приводятся результаты экспериментов по измерению электронной температуры с использованием мультиволновой лазерной методики, реализованной на токамаке Глобус-М2 [8,9]. В этих экспериментах для зондирования плазмы используются два импульсно-периодических лазерных источника на кристаллах Nd:YAG и Nd:YLF, излучающие на длинах волн 1064 и $1047 \mathrm{~nm}$ соответственно. Nd:YAG-лазер генерирует импульсы с энергией в каждом импульсе $\leqslant 3$ J при длительности импульса $10 \mathrm{~ns}$ с частотой следования импульсов до $300 \mathrm{~Hz}$. Для Nd:YLF-лазера энергия 

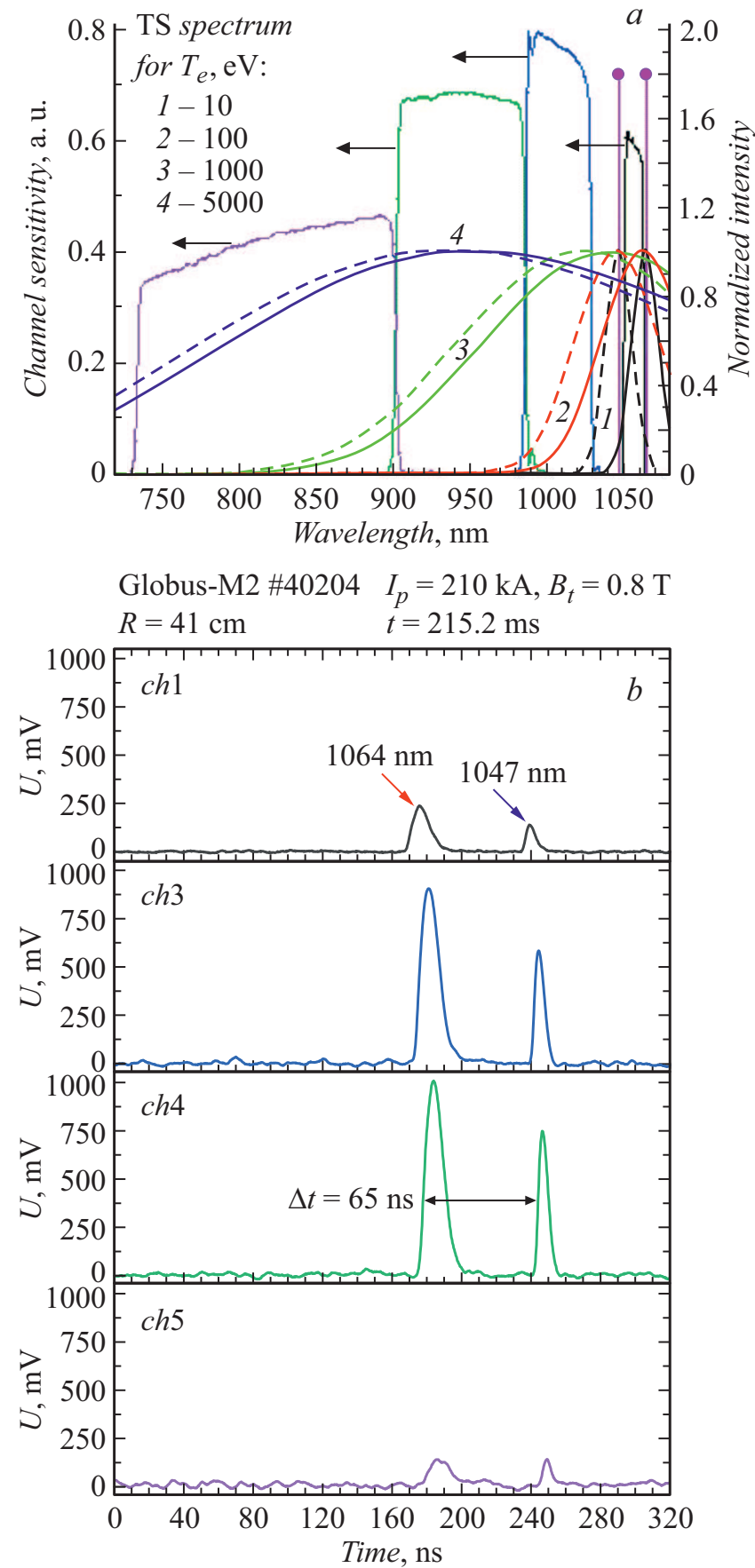

Рис. 1. $a-$ сравнение спектральной характеристики полихроматора с контурами рассеянного излучения для обоих значений зондирующих длин волн при различной температуре электронов. Сплошные кривые соответствуют контурам рассеяния для $\lambda_{01}=1064 \mathrm{~nm}$, штриховые - для $\lambda_{02}=1047 \mathrm{~nm}$. Положение зондирующих длин волн обозначено вертикальными маркерами. $b-$ осциллограммы синхронизованных сигналов томсоновского рассеяния для $\lambda_{01}=1064 \mathrm{~nm}$ и $\lambda_{02}=1047 \mathrm{~nm}$.

импульсов составляет до $2 \mathrm{~J}$ при длительности импульса $3 \mathrm{~ns}$ с частотой следования импульсов до $50 \mathrm{~Hz}$. Для регистрации сигналов рассеяния применялась система фильтровых спектрометров, оснащенная прецизионной системой регистрации [10,11]. Особенностью диагностической системы является возможность регулировки временно́й задержки между лазерными импульсами Nd:YAG- и Nd:YLF-лазеров, обеспечивающей синхронизацию лазерных импульсов с наносекундной точностью.

В предположении, что на временно́м масштабе $<100 \mathrm{~ns}$ локальные параметры плазмы в токамаке являются постоянной величиной, температуру электронов можно определить, минимизируя выражение [3]:

$$
\sum_{i=1}^{N} \frac{\left(S_{i}-\gamma F_{i}\left(T_{e}\right)\right)^{2}}{\sigma_{S_{i}}^{2}} \rightarrow \min .
$$

Здесь $\gamma$ - соотношение энергии импульсов лазеров в наблюдаемом рассеивающем объеме, $S_{i}$ - отношение сигналов $i$-го канала спектрометра, детектирующего рассеяние лазерного излучения на двух длинах волн $\lambda_{01}$ и $\lambda_{02}: S_{i}=U_{\lambda_{01}}^{\mathrm{TS}} / U_{\lambda_{02_{i}}}^{\mathrm{TS}}, \sigma_{S_{i}}^{2}-$ оценка дисперсии этого отношения, а $F_{i}\left(T_{e}\right)$ - ожидаемая зависимость отношения сигналов рассеяния от температуры электронов:

$$
F_{i}\left(T_{e}\right) \approx \frac{\int_{\lambda_{\min _{i}}}^{\lambda_{\max _{i}}} \sigma_{\mathrm{TS}}\left(T_{e}, \lambda, \lambda_{01}\right) d \lambda}{\int_{\min _{i}}^{\lambda_{\max _{i}}} \sigma_{\mathrm{TS}}\left(T_{e}, \lambda, \lambda_{02}\right) d \lambda} .
$$

Здесь $\lambda_{\min _{i}}$ и $\lambda_{\max _{i}}$ - границы соответствующего спектрального канала.

Демонстрация применения данного подхода в плазменном эксперименте была выполнена на модернизированной системе диагностики томсоновского рассеяния токамака Глобус-М2. Для этого существующая система зондирования была оснащена дополнительным лазером Nd:YLF с генерацией на длине волны $1047 \mathrm{~nm}$. Данный лазерный источник разрабатывался в качестве калибровочного лазера для диагностики диверторной плазмы токамака-реактора ИТЭР. Основной его задачей на ИТЭР является калибровка спектральной аппаратуры по сигналам ТР в узком диапазоне электронной температуры $<200 \mathrm{eV}[1]$. Длительность лазерного импульса на половине высоты в эксперименте составляла 3 ns с частотой следования $50 \mathrm{~Hz}$. Оптические затворы лазерных источников Nd:YAG и Nd:YLF были синхронизованы, каждый шестой импульс Nd:YAG-лазера, работавшего с частотой $300 \mathrm{~Hz}$, сопровождался импульсом $\mathrm{Nd}$ :YLF с временно́й задержкой 60-70 ns. Применение детекторов с высокой полосой пропускания и высокоскоростной системы регистрации 3.2 GSamples/s позволило записывать сигналы рассеяния от обоих лазеров в пределах одной страницы памяти оцифровщика.

Сравнение спектральной характеристики полихроматора с контурами рассеянного излучения, соответствующими разной температуре электронов и измеренными на обеих длинах волн зондирования, представлено на рис. 1,a. Поскольку фильтровая система полихроматоров ТР токамака Глобус-М2 не предназначена 

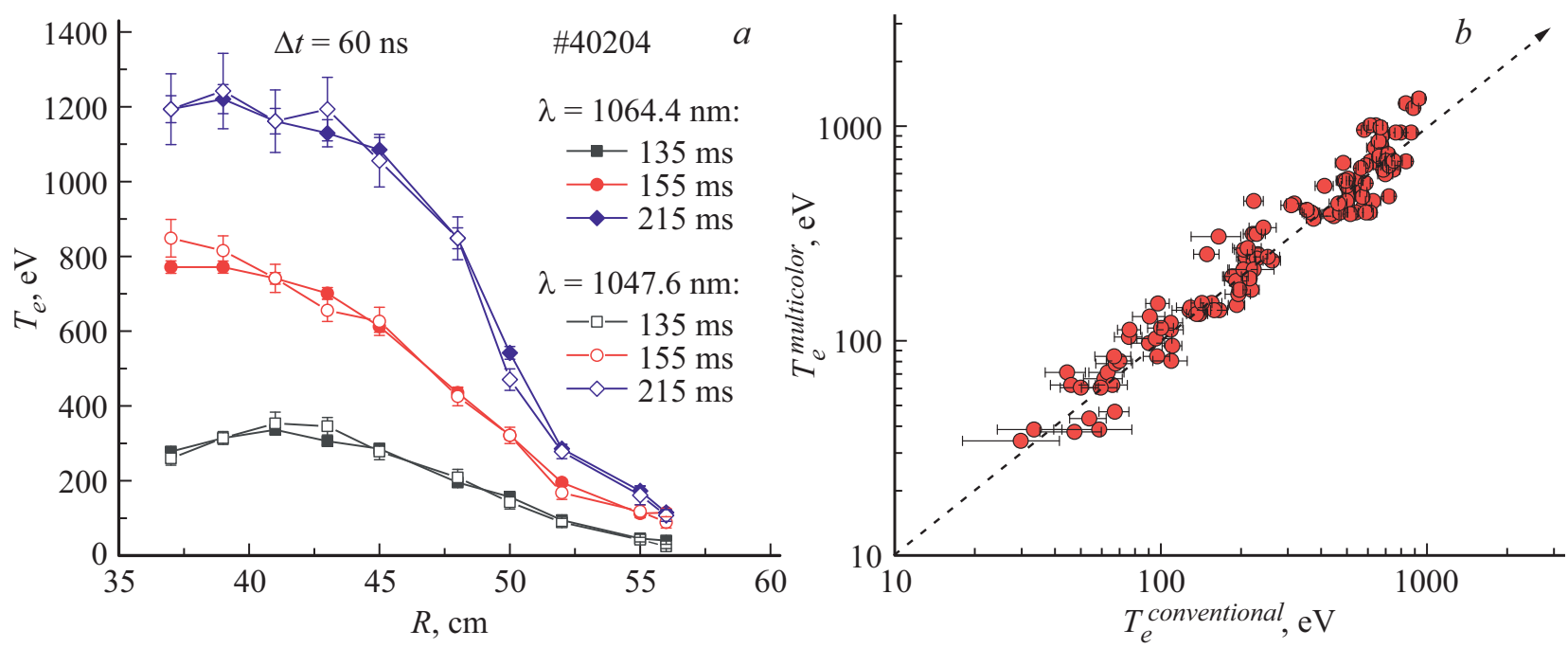

Рис. 2. $a-$ сравнение измеренных профилей температуры электронов в различные фазы разряда токамака Глобус-М 2 с временно́й задержкой 60-70 ns. $b$ - сравнение температуры электронов, рассчитанной с помощью мультилазерной методики в предположении неизвестной спектральной калибровки системы (ось ординат), со значениями, определенными традиционным способом (ось абсцисс).

для измерения сигналов ТР от лазера, излучающего на длине волны $1047 \mathrm{~nm}$, высокий уровень паразитнорассеянного излучения во втором спектральном канале привел к необходимости его отключения. Следует отметить, что величина $S_{1}$ практически не зависит от температуры электронов, так как критическая длина волны (см. [3]) приходится на первый спектральный канал: $\lambda_{\text {crit }}=\sqrt{\lambda_{01} \lambda_{02}}=1055 \mathrm{~nm}$. Из-за достаточно близкого расположения зондирующих длин волн измерение высоких температур с использованием мультилазерной методики требует высокой точности измерения сигналов рассеяния в коротковолновых спектральных каналах.

На рис. 1, $b$ приведены примеры осциллограмм синхронизованных сигналов томсоновского рассеяния. Первый по времени импульс соответствует лазеру 1064 nm, второй — лазеру $1047 \mathrm{~nm}$. Временна́я задержка между лазерными импульсами составляет величину 60-70 ns. На рис. 2, а показано сравнение измеренных в эксперименте соответствующих профилей температуры электронов. Расчет $T_{e}$ проводился на основании данных спектральной калибровки. Видно, что на данном временно́м масштабе расхождение измеренных значений $T_{e}$ не превышает оценку погрешности измерения.

Рис. 2, $b$ иллюстрирует сравнение температуры электронов, рассчитанной с помощью мультилазерной методики в предположении неизвестной спектральной калибровки системы (ось ординат), со значениями, определенными традиционным способом (ось абсцисс). Из приведенной зависимости видно, что мультилазерное зондирование позволяет достоверно определять температуру электронов. Для ответа на вопрос о том, позволит ли предлагаемый подход отследить возможное изменение спектральной характеристики системы сбора света, был проведен специальный эксперимент. Искаже- ние спектральной характеристики оптической системы сбора света как вследствие радиационно-наведенного поглощения $[2,4]$, так и вследствие загрязнения оптических элементов продуктами эрозии первой стенки [1] будет проявляться в первую очередь в виде снижения пропускания в более коротковолновой области спектра. Подобное искажение можно сымитировать с помощью цветного стекла ЖС-20, установленного в оптический тракт диагностики между объективом и оптоволоконными жгутами. Внесение такого изменения привело к аппаратному искажению контура рассеяния, вызванному пропусканием цветного стекла. На рис. 3, $a$ приведено сравнение неискаженного и искаженного контуров рассеяния для температуры электронов $1 \mathrm{keV}$. Видно, что в отсутствие информации об изменении спектральной характеристики системы традиционная диагностика ошибочно определяет температуру электронов как 0.5-0.6 keV (контуры, показанные штриховыми линиями на рисунке) вместо $1 \mathrm{keV}$. На рис. $3, b$ приведено сравнение температуры электронов, рассчитанной с помощью мультилазерной методики в предположении неизвестной спектральной калибровки системы (ось ординат), со значениями, определенными традиционным способом (ось абсцисс) для неискаженных спектральных характеристик. Из рисунка видно, что основная часть точек лежит выше прямой $x=y$, что указывает на систематическое занижение величины $T_{e}$, рассчитанной в рамках традиционного подхода, что было ожидаемо. Также, как и предполагалось, занижение величины $T_{e}$ тем больше, чем шире спектральный контур рассеянного излучения, т. е. чем выше значения самой электронной температуры. При истинном значении $T_{e}=1 \mathrm{keV}$ измерения, выполненные в рамках традиционного подхода, 

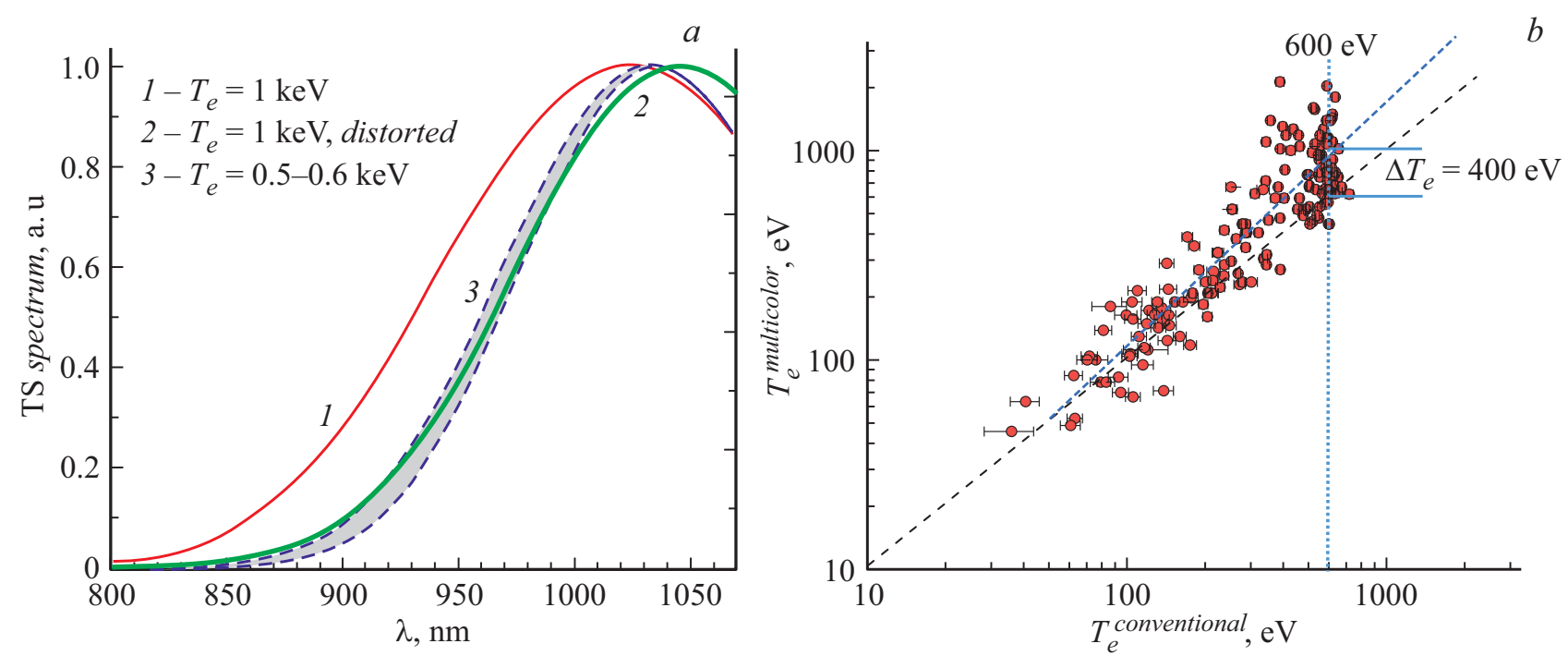

Рис. 3. $a-$ искажение спектрального контура рассеянного лазерного изучения для температуры электронов $1 \mathrm{keV}$ при внесении цветного стекла ЖС-20 в оптическую схему системы сбора света. $1-$ неискаженный контур для $T_{e}=1 \mathrm{keV}, 2-$ контур для $T_{e}=1 \mathrm{keV}$ после прохождения через цветное стекло, 3 - неискаженные контуры рассеянного лазерного изучения для $T_{e}=0.5$ и $0.6 \mathrm{keV} . b$ - зависимость температуры электронов (неизвестная спектральная калибровка системы) от значений, определенных традиционным способом для неискаженных спектральных характеристик.

оказались в соответствии с расчетами в среднем на $0.4 \mathrm{keV}$ ниже.

Таким образом, на токамаке Глобус-М2 были проведены измерения температуры электронов в диапазоне 30-1000 eV методом двухволнового томсоновского рассеяния. Временна́я задержка между зондирующими лазерными импульсами на длинах волн 1047 и $1064 \mathrm{~nm}$ составляла несколько десятков наносекунд. Продемонстрирована система томсоновского рассеяния, позволяющая проводить достоверные измерения температуры электронов в условиях неизвестной спектральной характеристики системы сбора рассеянного излучения. Созданная мультилазерная диагностическая система позволяет проводить измерения в стационарном режиме работы установки и может быть использована для применения в условиях термоядерного реактора и источника термоядерных нейтронов.

\section{Благодарности}

Работа выполнена на УНУ „Сферический токамак Глобус-М“, входящей в состав ФЦКП „Материаловедение и диагностика в передовых технологиях“.

\section{Финансирование работы}

Работа частично финансировалась в рамках государственного задания ФТИ им. А.Ф. Иоффе. Подготовка лазерной системы $\mathrm{Nd}$ :YLF $(1047 \mathrm{~nm})$ выполнена при частичной финансовой поддержке в рамках выполнения работ по договору № 17706413348210001850/29-21/01 от 28.05.2021 г.

\section{Конфликт интересов}

Авторы заявляют, что у них нет конфликта интересов.

\section{Список литературы}

[1] E.E. Mukhin, R.A. Pitts, P. Andrew, I.M. Bukreev, P.V. Chernakov, L. Giudicotti, G. Huijsmans, M.M. Kochergin, A.N. Koval, A.S. Kukushkin, G.S. Kurskiev, A.E. Litvinov, S.V. Masyukevich, R. Pasqualotto, A.G. Razdobarin, V.V. Semenov, S.Yu. Tolstyakov, M.J. Walsh, Nucl. Fusion, 54 (4), 043007 (2014). DOI: 10.1088/0029-5515/54/4/043007

[2] M. Bassan, P. Andrew, G. Kurskiev, E. Mukhin, T. Hatae, G. Vayakis, E. Yatsuka, M. Walsh, J. Instrum., 11 (1), C01052 (2016). DOI: 10.1088/1748-0221/11/01/C01052

[3] O.R.P. Smith, C. Gowers, P. Nielsen, H. Salzmann, Rev. Sci. Instrum., 68 (1), 725 (1997). DOI: 10.1063/1.1147686

[4] G.S. Kurskiev, P.A. Sdvizhenskii, M. Bassan, P. Andrew, A.N. Bazhenov, I.M. Bukreev, P.V. Chernakov, M.M. Kochergin, A.B. Kukushkin, A.S. Kukushkin, E.E. Mukhin, A.G. Razdobarin, D.S. Samsonov, V.V. Semenov, S.Yu. Tolstyakov, S. Kajita, S.V. Masyukevich, Nucl. Fusion, 55 (5), 053024 (2015). DOI: 10.1088/0029-5515/55/5/053024

[5] O. McCormack, L. Giudicotti, A. Fassina, R. Pasqualotto, Plasma Phys. Control. Fusion, 59 (5), 055021 (2017). DOI: $10.1088 / 1361-6587 /$ aa6692

[6] В.В. Солоха, Г.С. Курскиев, Е.Е. Мухин, С.Ю. Толстяков, А.Н. Баженов, Н.А. Бабинов, И.Н. Букреев, А.М. Дмитриев, М.М. Кочергин, А.Н. Коваль, А.Е. Литвинов, С.В. Масюкевич, А.Г. Раздобарин, Д.С. Самсонов, В.В. Семенов, В.А. Соловей, П.В. Чернаков, Ал.П. Чернаков, Ан.П. Чернаков, С.В. Иваненко, А.Д. Хильченко, Е.А. Пурыга, А.Н. Квашнин, в сб. XLIV Межсдународная (Звенигородская) конференция по физике плазмы и управляемому термоядерному синтезу (Звенигород, 2017), c. 98. 
[7] H. Tojo, A. Ejiri, J. Hiratsuka, T. Yamaguchi, Y. Takase, K. Itami, T. Hatae, Rev. Sci. Instrum., 83 (2), 023507 (2012). DOI: $10.1063 / 1.3685612$

[8] V.K. Gusev, E.A. Azizov, A.B. Alekseev, A.F. Arneman, N.N. Bakharev, V.A. Belyakov, S.E. Bender, E.N. Bondarchuk, V.V. Bulanin, A.S. Bykov, F.V. Chernyshev, I.N. Chugunov, V.V. Dyachenko, O.G. Filatov, A.D. Iblyaminova, M.A. Irzak, A.A. Kavin, G.S. Kurskiev, S.A. Khitrov, N.A. Khromov, V.A. Kornev, S.V. Krasnov, E.A. Kuznetsov, A.N. Labusov, M.M. Larionov, K.M. Lobanov, A.A. Malkov, A.D. Melnik, V.B. Minaev, A.B. Mineev, M.I. Mironov, I.V. Miroshnikov, A.N. Novokhatsky, A.D. Ovsyannikov, A.A. Panasenkov, M.I. Patrov, M.P. Petrov, Yu.V. Petrov, V.A. Rozhansky, V.V. Rozhdestvensky, A.N. Saveliev, N.V. Sakharov, P.B. Shchegolev, O.N. Shcherbinin, I.Yu. Senichenkov, V.Yu. Sergeev, A.E. Shevelev, A.Yu. Stepanov, V.N. Tanchuk, S.Yu. Tolstyakov, V.I. Varfolomeev, A.V. Voronin, F. Wagner, V.A. Yagnov, A.Yu. Yashin, E.G. Zhilin, Nucl. Fusion, 53 (9), 093013 (2013). DOI: 10.1088/0029-5515/53/9/093013

[9] V.B. Minaev, V.K. Gusev, N.V. Sakharov, V.I. Varfolomeev, N.N. Bakharev, V.A. Belyakov, E.N. Bondarchuk, P.N. Brunkov, F.V. Chernyshev, V.I. Davydenko, V.V. Dyachenko, A.A. Kavin, S.A. Khitrov, N.A. Khromov, E.O. Kiselev, A.N. Konovalov, V.A. Kornev, G.S. Kurskiev, A.N. Labusov, A.D. Melnik, A.B. Mineev, M.I. Mironov, I.V. Miroshnikov, M.I. Patrov, Yu.V. Petrov, V.A. Rozhansky, A.N. Saveliev, I.Yu. Senichenkov, P.B. Shchegolev, O.N. Shcherbinin, I.V. Shikhovtsev, A.D. Sladkomedova, V.V. Solokha, V.N. Tanchuk, A.Yu. Telnova, V.A. Tokarev, S.Yu. Tolstyakov, E.G. Zhilin, Nucl. Fusion, 57 (6), 066047 (2017). DOI: 10.1088/1741-4326/aa69e0

[10] G.S. Kurskiev, Al.P. Chernakov, V.A. Solovey, S.Yu. Tolstyakov, E.E. Mukhin, A.N. Koval, A.N. Bazhenov, S.E. Aleksandrov, N.S. Zhiltsov, V.A. Senichenkov, A.V. Lukoyanova, P.V. Chernakov, V.I. Varfolomeev, V.K. Gusev, E.O. Kiselev, Yu.V. Petrov, N.V. Sakharov, V.B. Minaev, A.N. Novokhatsky, M.I. Patrov, A.V. Gorshkov, G.M. Asadulin, I.S. Bel'bas, Nucl. Instrum. Meth. Phys. Res. A, 963, 163734 (2020). DOI: 10.1016/j.nima.2020.163734

[11] N.S. Zhiltsov, G.S. Kurskiev, E.E. Mukhin, V.A. Solovey, S.Yu. Tolstyakov, S.E. Aleksandrov, A.N. Bazhenov, A1.P. Chernakov, Nucl. Instrum. Meth. Phys. Res. A, 976, 164289 (2020). DOI: 10.1016/j.nima.2020.164289 